\title{
Effect of structuring the workspace on cognitive and sensorimotor distance estimation: No dissociation between perception and action
}

\author{
YANN COELLO and ORIANNE IWANOW \\ Université Charles de Gaulle, Lille, France
}

\begin{abstract}
Independent processing of visual information for perception and action is supported by studies about visual illusions, which showed that context information influences overt judgment but not reaching attempts. The objection was raised, however, that these two types of performance are not directly comparable, since they generally focus on different properties of the visual input. The goal of the present study was to quantify the influence of context information (in the form of a textured background) on the cognitive and sensorimotor processing of egocentric distance. We found that the subjective area comprising reachable objects (probed with a cognitive task) decreased, whereas the amplitude of reaching movement (probed with a sensorimotor task) increased in the presence of the textured background with both binocular and monocular viewing. Directional motor performance was not affected by the experimental conditions, but there was a tendency for the kinematic parameters to mimic trajectory variations. The similar but opposite effects of the textured background in the cognitive and sensorimotor tasks suggested that in both tasks the visual targets were perceived as closer when they were presented in a sparse environment. A common explanation for the opposite effects was confirmed by the percentage of background influence, which was highly correlated in the two tasks. We conclude that visual processing for perception and action cannot be dissociated from context influence, since it does not differ when the tasks entail the processing of similar spatial characteristics.
\end{abstract}

In order to modify the predisposition of our sensory organs and motor effectors appropriately in relation to the environment, the central nervous system must select just those inputs that are currently relevant while suppressing irrelevant inputs (Allport, 1989; Desimone \& Duncan, 1995; Mattingley \& Driver, 1997; Tipper, Lortie, \& Baylis, 1992). In the past decade, a fascinating debate about how such selection occurs has been in the scientific foreground, with a particular focus on the perceptual and motor aspects of behavior. In this respect, neurophysiological, neuropsychological, and psychophysical data have provided converging evidence of distinct processing of spatial information within the visual system, depending on whether the task calls for object recognition, description, or visually guided reaching movement (Milner \& Goodale, 1995; Pagano \& Bingham, 1998; Schneider, 1969; Trevarthen, 1968; Ungerleider \& Mishkin, 1982). The observation of visual illusions led to the formation of psychophysical arguments for such dissociation, in which metric judgment of the central part of a visual pattern (e.g., the Müller-Lyer, Ponzo, or Titchner illusion) or of its location (e.g., the induced Roelofs effect) was influenced by context information during verbal responding but not

Correspondence concerning this article should be addressed to Y. Coello, Laboratoire URECA, Université Charles de Gaulle, Lille, BP 60149, F. 59653 Villeneuve d'Ascq Cedex, France (e-mail: yann .coello@univ-lille3.fr). during manual reaching (Rossetti, 1998). In an attempt to consider the latter visual illusion more precisely, healthy adults were asked to estimate the position of a luminous target appearing inside a surrounding frame centered according to the egocentric straight-ahead direction or with a lateral offset of $\pm 5^{\circ}$ in the frontoparallel plane (Bridgeman, 1991). When the frame was presented with a lateral offset, target location was misperceived as being in the opposite direction when estimated through verbal responses (which are elicited to probe cognitive processing), but not when estimated with manual reaching (which is used to probe sensorimotor processing). This influence of context information was thought to result from the fact that the visual system selects spatial characteristics in different ways depending on the output (Bridgeman, 1991, 2000). The purpose of visual processing for cognitive purposes, which deals with relative positions, is to give access to an explicit qualitative encoding of the visual space, including the processing of information relating to the whole visual scene, even when the influence of contextual elements leads to localization errors. This encoding serves as the basis for categorization and verbal report (e.g., determining if one object is circular or is above another object; Kosslyn, 1994). Conversely, the purpose of visual processing for sensorimotor purposes, which has to do with absolute positions, is to give access to an implicit quantitative (metric) encoding of the visual space that is insensitive to context information and serves as a guide for goal-directed behavior. The dissociation observed with the 
induced Roelofs effect was taken as evidence of distinct processing of visual information depending on whether the purpose of the task is cognitive or sensorimotor, since only the verbal account of target location was sensitive to the perturbing contextual frame (Bridgeman, 1991, 2000; Jeannerod \& Rossetti, 1993; Paillard, 1987). The observation that the visual system is anatomically organized into two main streams projecting from the visual cortex (V1) to the inferotemporal cortex (the ventral stream) and to the posterior parietal cortex (the dorsal stream) has naturally served as a general framework that supported the distinct use of spatial information depending on the constraints of the task (for a review, see Milner \& Goodale, 1995; Rossetti, 1998).

However, recent psychophysical data cast some doubt on the validity of this widespread view by showing that motor performance can be strongly influenced by visual context when various spatial dimensions can be differentiated. In particular, when participants pointed to a visual target in the absence of dynamic visual feedback, their distance performance was found to be much more accurate when the target was presented on a textured background than when it appeared on a nonperceptible one (Coello, Magne, \& Plenacoste, 2000; Conti \& Beaubaton, 1980; Foley \& Held, 1972; Tresilian, Mon-Williams, \& Kelly, 1999) and when the target was presented within a large rather than a restricted visual field (Bingham, 1993; Coello \& Grealy, 1997). The location of context information in relation to the self and the target also played a crucial role in determining reaching accuracy, with elements placed in the space through which the reach occurs conferring the most benefit (Coello, 2002; Grealy, Coello, \& Heffernan, 2003). We recently reported that the unexpected provision of a textured background in the action space has an instantaneous concomitant effect on movement amplitude and early kinematic characteristics such as peak velocity (Magne \& Coello, 2002), suggesting that the improvement of motor performance was mainly the consequence of a more accurate visual system. Interestingly, the conspicuous effect of structuring the visual space on distance accuracy usually left direction performance unaffected (Magne \& Coello, 2002).

This parametric framework has proved to have great implications in the debate relating to the perception-action dichotomy (Milner \& Goodale, 1995). Using the induced Roelofs effect (Bridgeman, 1991) in slightly different experimental conditions, we found that the presence of the off-center frame can influence motor responses in a way similar to that of perceptual reports, depending on the spatial dimension tested (Coello, Richaud, Magne, \& Rossetti, 2003). When the frame was displaced along the frontoparallel axis, the visual target was perceived as shifted in the direction opposite that of the off-center frame, but the manual capture of the target was not affected by the illusion, in agreement with the original studies (Bridgeman, 1991, 2000; Bridgeman, Peery, \& Anand, 1997). However, in contrast with the previous finding, the induced Roelofs effect interfered with perceptual and motor responses in identical ways when the frame was displaced along the sagittal axis. Our interpretation of the observation that the dissociated effect on motor performance depends on the dimension tested was that target location for action is not always immune from contextual influence, which really depends on the type of visual information that needs to be processed according to the task constraints. Because distance coding is strongly influenced by the visual cues available on a large part of the retina (Magne \& Coello, 2002), especially those lying within the effector-to-target gap that delimits action space (Coello, 2002), it was not surprising to observe that context information can influence motor production when the latter is performed in the near-far rather than the right-left dimension (Coello et al., 2003). The fact that a similar pattern of results was subsequently obtained with the patient (I.G.) suffering from optic ataxia due to bilateral damage of posterior parietal cortex indicates that the integrity of the dorsal pathway, in particular the superior parietal lobe, is not a necessary condition to obtain an influence of visual context on motor acts (Coello \& Rossetti, 2004).

Although these findings directly challenge a radical separation between perception and action on the basis of the distinct influence of context information, one must consider them cautiously and cannot conclude straightaway that the visual system can be influenced by context information in similar ways for perception and for action. Indeed, just as in many previous studies dealing with visual illusions (see, e.g., Franz, Gegenfurtner, Bülthoff, \& Fahle, 2000), the perceptual and motor tasks in the adapted version of the induced Roelofs effect required the processing of different kinds of spatial information. In the perceptual task, participants had to estimate the relative position of the visual target with regard to another target presented $500 \mathrm{msec}$ earlier. The first target (400 msec in duration) was always visible within a center frame, whereas the second target (400 msec in duration) was presented within an off-center frame or without any frame. By contrast, the motor performance consisted of a pointing movement toward the second target with no need to explicitly process the previous one (see Coello et al., 2003, for a detailed description). It thus sufficed to estimate the egocentric absolute location of the last visible target in order to perform the task accurately. Consequently, one cannot firmly conclude from these data that context information can influence the perceptual and motor responses similarly when the task emphasizes the processing of a distance parameter.

To unravel this issue, it was necessary to compare the cognitive and sensorimotor visual systems in a situation requiring the processing of the same kind of spatial information, as well as to quantify the respective influence of context information. To meet these requirements, the influence of visual context (in the form of a textured background) was investigated in two tasks involving the processing of the absolute egocentric distance of a visual target. In the cognitive task, participants had to provide an overt perceptual judgment about whether or not the 
visual target was reachable with the hand, but with no actual movement allowed. Several studies have shown that people are quite accurate in estimating perceptually the critical limit of what is reachable. The general agreement is that one's estimation of one's own reaching capabilities exceeds actual arm length by about 10\% (Bootsma, Bakker, van Snippenberg, \& Tdlohreg, 1992; Carello, Grosofsky, Reichel, Solomon, \& Turvey, 1989; Rochat \& Wraga, 1997). In the sensorimotor task, in a dynamic open-loop condition, participants had to perform pointing movements toward a visual target located at various distances along the sagittal axis. Assuming that the cognitive and sensorimotor visual systems are similarly influenced by context information, one would expect the effect of the textured background to be of the same magnitude on verbal and motor responses but opposite in direction. Indeed, assuming that reaching movements are less hypometric in the presence of a textured background than in its absence, because visual objects appear nearer in a sparsely structured environment (Coello et al., 2003; Foley, 1991; Watt, Bradshaw, \& Rushton, 2000), the farthest target judged as reachable in darkness should be judged as not reachable in the presence of a textured background.

\section{METHOD}

\section{Participants}

The present study involved 8 normal right-handed participants ( 3 males and 5 females). Their ages ranged from 16 to 35 years, and they were self-declared volunteers for an experiment relating to visual perception and visuomotor control. They all had normal or corrected-to-normal vision (the right eye was systematically the dominant eye) and were naive as to the purpose of the experiment.

\footnotetext{
Apparatus

The experimental device consisted of a rectangular box $(60 \mathrm{~cm}$ high $\times 100 \mathrm{~cm}$ wide $\times 70 \mathrm{~cm}$ deep) with one side left open (see Figure 1). The inside of the box was divided horizontally by an upward-facing reflecting mirror. With the participant's head resting on the upper part of the box on the open side, only the top half of the box was visible, and the participant was able to move the right arm into the bottom half when requested to point to a visual target. A computer monitor (Philips 20-in. Trinitron) was placed upside down on the top surface of the apparatus so that the image generated by the computer was reflected in the mirror. Due to optical geometry, the image of the computer screen projected onto the bottom surface of the box - that is, onto the workspace. The mirror was positioned so that the hand was visible at the starting position but was concealed by the mirror following movement onset. Several visual targets (8-mm-diam green dots) were visible along the sagittal plane, each of them being presented alone (background $0 \mathrm{~cd} / \mathrm{m}^{2}$, measured with the Cambridge Research System optical photometer) or on a textured background made by $5-\mathrm{mm}\left(4-\mathrm{cd} / \mathrm{m}^{2}\right)$ gray dots randomly positioned over the entire workspace $(30 \times 39 \mathrm{~cm})$. To determine the random position of the texture dots, the following procedure was used. Interdot distance was initially fixed (one dot every $15 \mathrm{~mm}$ in the $x$ - and $y$-directions), but before the visual information was displayed a random coefficient was assigned by the computer to each of the targets so that their $(x, y)$ position corresponded to a random value comprised between $\pm 0 \%$ and $\pm 50 \%$ of the fixed interdot distance. Dots could not overlap, and their positions were recomputed at the beginning of each trial. The number and locations of the targets varied as a function of the experimental condition, as described below.
}

Two target displays were used: one for the cognitive task and one for the sensorimotor task. In the cognitive task, 21 visual targets were selected along the sagittal axis at distances corresponding to the individual participant's maximum distance reachable with the hand (Target 0 in Figure 1 ) and at $\pm 5, \pm 10, \pm 15, \pm 20, \pm 25, \pm 30, \pm 35$, $\pm 40, \pm 45$, and $\pm 50 \mathrm{~mm}$ from that position (Targets +10 to -10 ). The maximum reachable distance (critical boundary) was initially estimated within the experimental box for each participant by measuring the distance that he or she could reach with the right arm fully extended. In the pointing task, only three targets were used along the sagittal axis. They were positioned 45,75 , and $105 \mathrm{~mm}$ nearer than the maximum reachable distance (Targets $-9,-15$, and -21 , respectively; see Figure 1). These distances were chosen so that they would be easily reachable but would account for significant variability along the sagittal axis. In order to minimize the participant's ability to use visual cues in the form of reflections from the upper half or the side of the box, the internal surfaces of the box were smoothed and painted matte black. No visual information from the external environment was available during the entire experimental session.

\section{Procedure}

During a familiarization period, the participants were taught to move an electromagnetic stylus horizontally on a digitizer (Wacom UD-1825; sample rate, $100 \mathrm{~Hz}$ ) so that its final position coincided with that of the visual target (sensorimotor task), or to give an overt perceptual judgment of whether or not the target was reachable (cognitive task). For each trial, the computer sequence was triggered when the electromagnetic stylus was positioned on the starting area, about $23 \mathrm{~cm}$ in front of the forehead plane on the sagittal axis. Following the contact of the stylus with the starting area, a target appeared either in isolation or together with a textured background following a random latency of $0-500 \mathrm{msec}$. After becoming familiarized with the various visual scenes (darkness or textured background) and with the various response conditions (verbal or pointing response) during about $15 \mathrm{~min}$ to allow for visual adaptation to the dark room, the participants began with one of the experimental tasks in a monocular or binocular vision condition. In the cognitive task, the 21 targets were presented five times in random order, whereas in the pointing task the 3 targets were presented eight times each in random order. The order of presentation of the task (cognitive or sensorimotor), the visual scene (darkness or textured background), and the vision condition (binocular or monocular) were counterbalanced across participants. The experiment was conducted without feedback for the participants about performance accuracy.

Data recording and processing. In the cognitive task, the verbal response was recorded online by the experimenter on a Macintosh iBook laptop. For each visual scene and for each vision condition, the critical limit of what was reachable was determined using a maximum likelihood fit procedure based on the second-order derivatives (quasi-Newton method) to obtain the logit regression model that best fit the yes/no responses of the participant for the 21 positions of the target, from the following equation:

$$
y=e^{(\alpha+\beta x)} /\left(1+e^{(\alpha+\beta x)}\right),
$$

where $y$ is the subject's response, $x$ is the distance, $-\alpha / \beta$ is the critical value of $x$ at which the transition from one type of response to the other occurs (thus expressing the subjective maximum distance that can be reached), and $\beta / 4$ is a measure of the slope at point $-\alpha / \beta$. A greater slope indicates an easier separation between "yes" and "no" responses for the participant.

In the sensorimotor task, the $x$ - and $y$-coordinates of the trajectory were registered from the digitizer tablet with a spatial resolution of $0.1 \mathrm{~mm}$. Endpoint positions of individual movements were used to compute constant and variable terminal error. In relation to our working hypothesis, constant errors were decomposed into radial (performance in amplitude) and angular (performance in direction) values. Radial error was evaluated from the distance between move- 


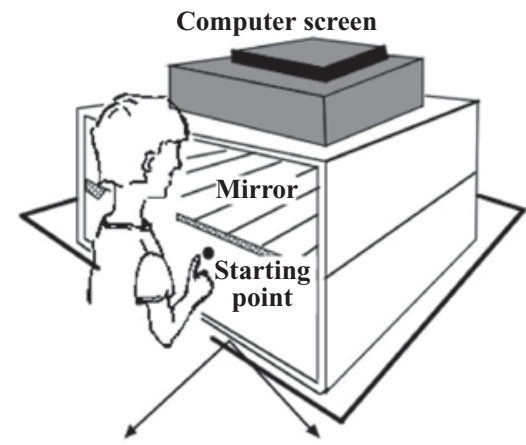

Cognitive Task

Sensorimotor Task

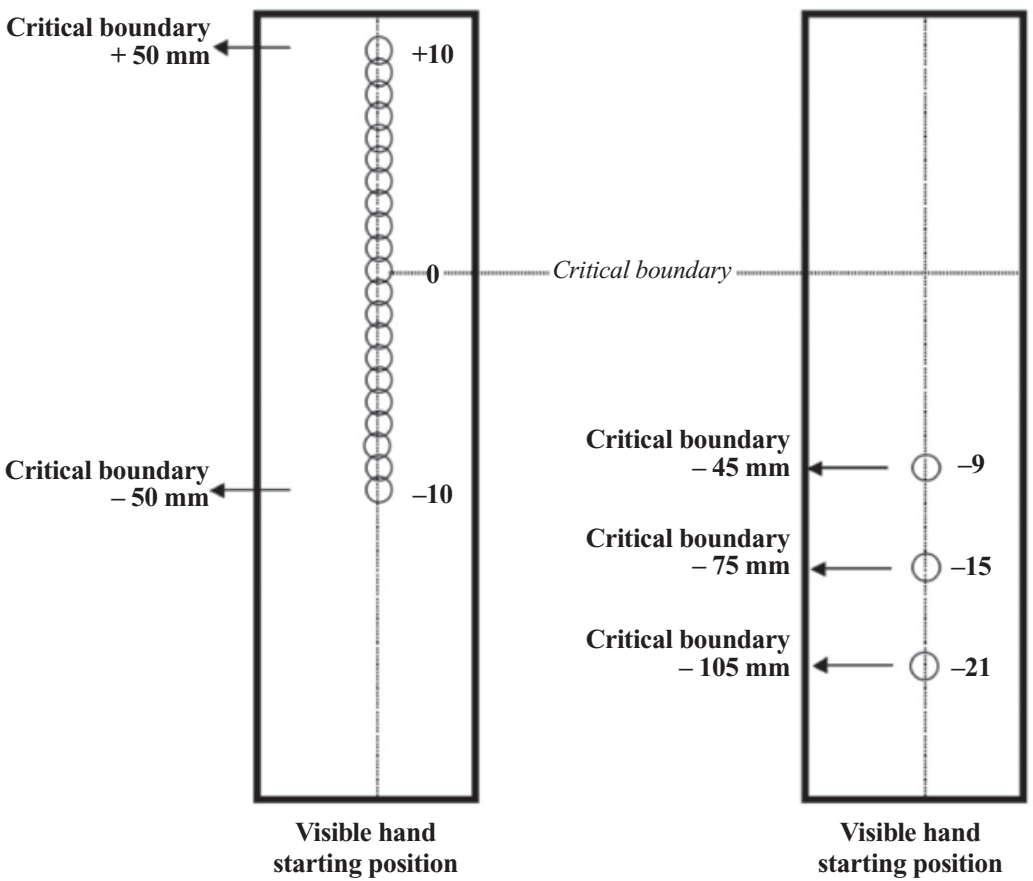

Figure 1. Schematic representation of the experimental apparatus and the layout of the visual targets used in the cognitive and sensorimotor tasks. The critical boundary corresponded to maximum arm length, measured individually for each participant.

ment vector length and target vector length (a minus sign [-] was used for undershoot, and a plus sign $[+]$ in the absence of undershoot). Angular error corresponded to the angle between the startingposition-to-target vector and the starting-position-to-end-movementposition vector (a minus sign was used for deviations to the right of the target, and a plus sign for other deviations or no deviation). Kinematic (i.e., peak velocity) and temporal (i.e., movement time and percentage of time taken by the acceleration and deceleration periods) parameters were also examined from hand path.

Statistical analyses were initially carried out separately for the cognitive and the sensorimotor tasks through a two-way ANOVA with visual scene (darkness vs. textured background) and vision condition (binocular vs. monocular) as factors, with repeated measures to test for main effects of visual scene and vision condition. Data relating to the various target positions were pooled for statistical investigations, which were carried out on all dependent variables. In the case that the sphericity assumption was violated (i.e., $\varepsilon<1$ ), Huynh-Feldt adjustments of the $p$ values were reported. The goal of this analysis was to evaluate whether or not context information influenced both the cognitive and the sensorimotor processing, but in opposite directions. We then compared the verbal and motor responses by comparing the relative effects (in percentages) of the textured background on distance performance, to determine whether or not they were similar in magnitude for every participant. For this, we computed, in the monocular and binocular vision conditions, the absolute difference between the results obtained in the textured background condition and those obtained in the darkness condition, divided by the reached distance $* 100$ (percentage of relative effect). In the cognitive task, this corresponded to $100 * \mid$ critical limit $_{\text {textured }}$ background - critical limit $_{\text {darkness }} \mid$ /critical limit ${ }_{\text {textured background. In the }}$ sensorimotor task, this corresponded to $100 * \mid$ amplitude performance $_{\text {textured background }}-$ amplitude performance ${ }_{\text {darkness }} \mid$ /amplitude

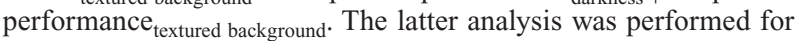
the farthest target only, since it was closer to the critical limit of what is reachable in the cognitive task. Finally, we computed the regression coefficient $(r)$ by measuring the degree of linearity between the relative textured background effect in the cognitive task and that in the sensorimotor tasks for all the participants. 


\section{RESULTS}

Mean arm length, measured as the distance between the shoulder joint (about $45 \mathrm{~cm}$ above the workspace) and the tip of the index finger while the arm is extended, was $67.25 \mathrm{~cm}$. Thus, the mean maximum reachable distance (critical boundary) on the workspace was $50 \mathrm{~cm}$ from the body and $27 \mathrm{~cm}$ from the starting position of the hand along the sagittal axis.

\section{Cognitive Coding of Target Location}

Estimated from the critical value $(-\alpha / \beta)$ of the logit regression, the limit of what is reachable as judged by the participants was $6.5 \mathrm{~mm}$ further than the critical boundary on average (see Figure 2A). However, this limit was farther in the darkness condition $(13.88 \mathrm{~mm})$ than in the textured background condition $[-0.91 \mathrm{~mm} ; F(1,7)=$ $11.82, p=.01]$. No effect of whether the vision was monocular $(6.84 \mathrm{~mm})$ or binocular $(6.13 \mathrm{~mm})$ was observed $[F(1,7)=0.04, p>.05]$. We also found no interaction between the two factors $[F(1,7)=0.81, p>.05]$. As is shown in Figure 2B, the influence of the textured background on the overt judgment of what is reachable was observed for all the participants, but with some interindividual differences concerning the effect of viewing condition (monocular vs. binocular).

The slope $(\beta / 4)$ of the logit regression, expressing the level of uncertainty in the cognitive decision, was 0.068 on average. It was not affected by the visual scene $[F(1,7)=$ $4.06, p>.05$, with 0.077 and 0.06 for the textured background and darkness conditions, respectively] or by the vision condition $[F(1,7)=0.47, p>.05$, with 0.064 and 0.073 for the binocular and monocular conditions, respectively]. No interaction between the two factors was observed $[F(1,7)=0.98, p>.05]$. Thus, the cognitive decision was not more difficult to make in any of the experimental conditions.

\section{Sensorimotor Coding of Target Location}

Radial error. Movement amplitude (see Figure 3A) was characterized by a substantial undershoot of target location ( $-37.86 \mathrm{~mm}$ on average), which corresponded to $21 \%$ of the target distance $(25 \%, 21 \%$, and $19 \%$ for targets located 105,75 , and $45 \mathrm{~mm}$ from the critical boundary, respectively). However, radial error was greater in darkness $(-46.38 \mathrm{~mm})$ than with the textured background $[-29.35 \mathrm{~mm} ; F(1,7)=18.72, p<.01]$ and greater in the monocular $(-44.53 \mathrm{~mm})$ than in the binocu$\operatorname{lar}(-31.21 \mathrm{~mm})$ condition $[F(1,7)=12.33, p<.01]$. A significant interaction between the two factors $[F(1,7)=$ $8.42, p=.02]$ showed that the target's undershoot was greater in the monocular $(-56.37 \mathrm{~mm})$ than in the binocular $(-36.40 \mathrm{~mm})$ condition when the textured background context was missing $[t(7)=6.16, p<.01]$ but not when it was available $[-32.69$ and $-26.01 \mathrm{~mm}$, respectively; $t(7)=2.06, p>.05]$. Radial error was lower with than without the textured background in both the monocular $[t(7)=7.31, p<.01]$ and the binocular $[t(7)=3.21, p=$ $.01]$ conditions, however. As is shown in Figure 3B, better distance accuracy was observed for all the participants in the presence of the textured background, with only 2 of the 8 participants showing no clear degradation of performance in the monocular vision condition.

Angular error. As was expected considering the parametric theoretical framework presented in the introduction, no effect of structuring the workspace on direction performance was observed (see Figure 4). The average angular error was $0.59^{\circ}$ to the left of the target both with and without the textured background $[F(1,7)=0.01, p>$ $.05]$. The binocular $\left(1.01^{\circ}\right)$ and monocular $\left(0.17^{\circ}\right)$ condi-
A

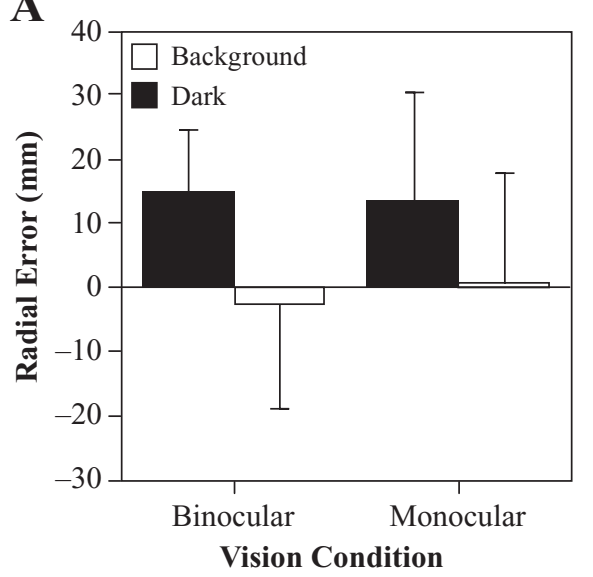

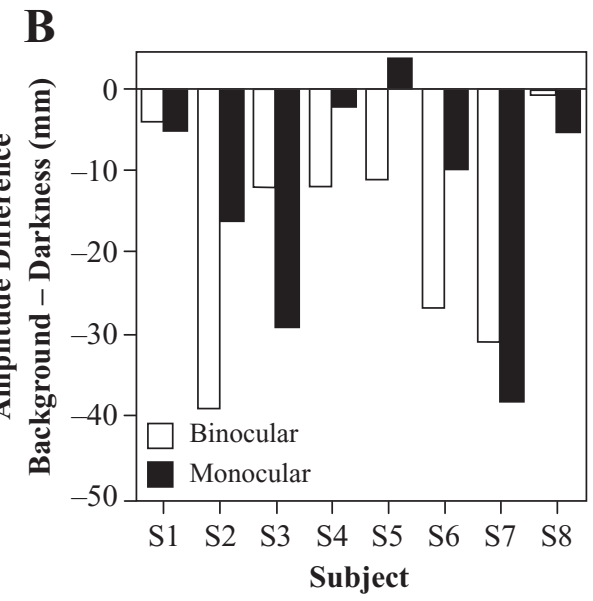

Figure 2. (A) Radial error and between-subjects variability in the cognitive task when the visual target was presented with and without a textured background in the binocular and monocular vision conditions. The 0 value corresponds to arm length. (B) Individual differences of radial error between the darkness condition and the textured background condition for the monocular and binocular vision conditions. Note that, for all the participants, the critical limit of what is reachable was perceived as further with the background than in darkness. 
A

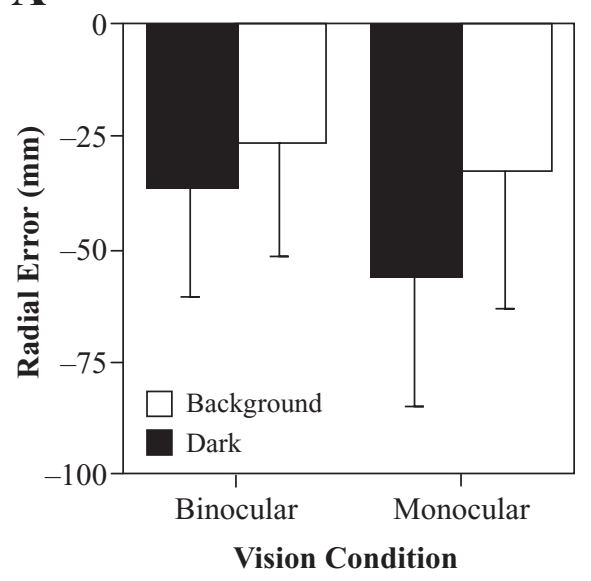

B

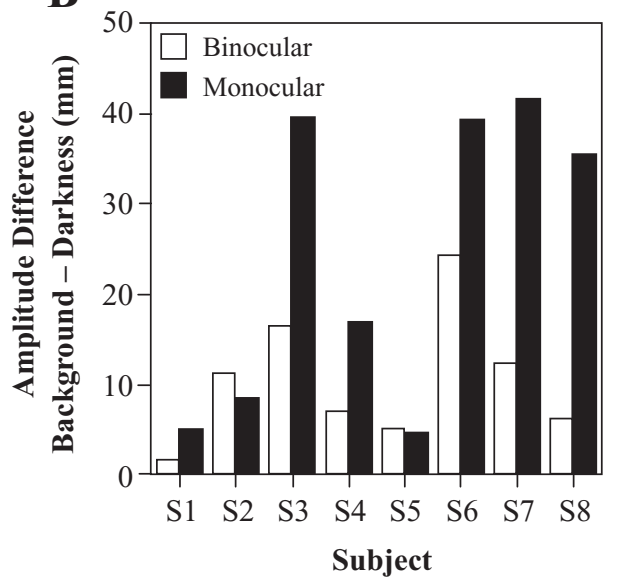

Figure 3. (A) Radial error and between-subjects variability in the sensorimotor task when the visual target was presented with and without a textured background in the binocular and monocular vision conditions. The results for the three targets have been pooled, and the 0 value corresponds to target location. (B) Individual differences for radial error between the darkness condition and the textured background condition in the monocular and binocular vision conditions. Note that, for all the participants, the extent of movement increased in the presence of a textured background.

tions also did not differ $[F(1,7)=0.74, p>.05]$, and no interaction between the two factors was noted $[F(1,7)=$ $0.04, p>.05]$.

Movement time. The pattern of results concerning movement time (552 $\mathrm{msec}$ on average) followed quite closely that of amplitude performance, in that it was greater with $(567 \mathrm{msec})$ than without $(539 \mathrm{msec})$ the textured background, although the statistical analysis showed only a tendency toward this effect $[F(1,7)=3.71, p=$ .09]. By contrast, the difference between the monocular $(539 \mathrm{msec})$ and binocular $(566 \mathrm{msec})$ vision conditions was highly significant $[F(1,7)=7.22, p=.03]$. No interaction between the two factors was noted $[F(1,7)=0.80$, $p>.05]$.

Peak velocity. The pattern of results concerning peak velocity $(538 \mathrm{~mm} / \mathrm{sec}$ on average) also showed only a tendency to follow the pattern of amplitude performance, in that it was greater in the presence of a textured background $(560 \mathrm{~mm} / \mathrm{sec})$ than with a dark visual scene $(516 \mathrm{~mm} /$ $\mathrm{sec}$ ), but the statistic computed value was slightly below the critical threshold $[F(1,7)=4.26, p=.07]$. The same analysis holds for the effect of visual condition, since peak velocity in the binocular condition $(551 \mathrm{~mm} / \mathrm{sec})$ was slightly higher than that in the monocular condition $(526 \mathrm{~mm} / \mathrm{sec})$, the difference being again close to significance $[F(1,7)=3.90, p=.08]$. The interaction between the two factors, however, was significant $[F(1,7)=5.64$, $p<.05]$ and resulted from the fact that peak velocity was greater in the monocular $(488 \mathrm{~mm} / \mathrm{sec})$ than in the binocu$\operatorname{lar}(545 \mathrm{~mm} / \mathrm{sec})$ condition when the textured background was not present [ $564 \mathrm{~mm} / \mathrm{sec} ; t(7)=2.70, p=.03]$, but not when it was present $[556 \mathrm{~mm} / \mathrm{sec} ; t(7)=0.35, p>$ $.05]$.
Acceleration and deceleration phase duration. The relative times taken by the acceleration and deceleration phases were estimated by analyzing the variation of the percentage of duration of the acceleration period with respect to movement time. In contrast with what was reported for movement time and peak velocity, the visual aspect of the workspace and the viewing conditions did not influence the intrinsic temporal organization of the movement. Indeed, the percentage of time taken by the ac-

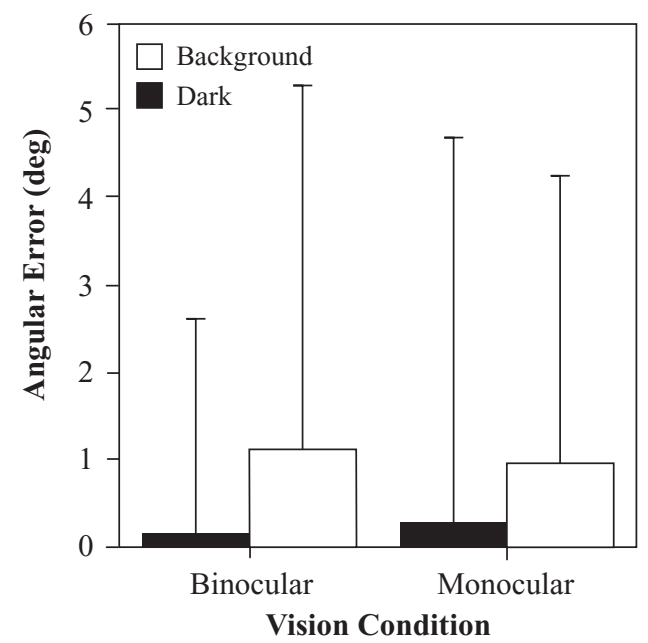

Figure 4. Angular error and between-subjects variability in the sensorimotor task when the visual target was presented with and without a textured background in the binocular and monocular vision conditions. The results for the three targets have been pooled, and the 0 value corresponds to the target location. 
celeration period ( $53 \%$ on average) was not influenced by the presence of the textured background $[F(1,7)=4.04$, $p>.05$, with $52 \%$ and $54 \%$ with and without the textured background, respectively] or by the vision condition $[F(1,7)=0.15, p>.05$, with $53 \%$ for both the monocular and binocular conditions]. Moreover, we found no interaction between the two factors $[F(1,7)=0.31, p>.05]$.

\section{Comparison of Cognitive and Sensorimotor Performance}

In order to compare cognitive performance and sensorimotor performance, we analyzed for the cognitive and the sensorimotor tasks the relative effect of providing the textured background in the visual scene by computing the absolute difference between the darkness condition and the textured background condition divided by the distance considered in the presence of the textured background (and the pointing distance in the sensorimotor task and the critical limit of what is reachable in the cognitive task). Knowing that the effects of structuring the workspace were opposite in sign in the cognitive and sensorimotor tasks (see above), we ought to establish whether the magnitude of the effect was similar in both tasks, and, if so, whether this was the case for all the participants.

Depicted in Figure 5 is the percentage of textured background influence in the cognitive and sensorimotor tasks for the binocular and monocular vision conditions. It is obvious from the data that the textured background had similar influence in the cognitive and sensorimotor tasks, since for every participant the scores in the two tasks were
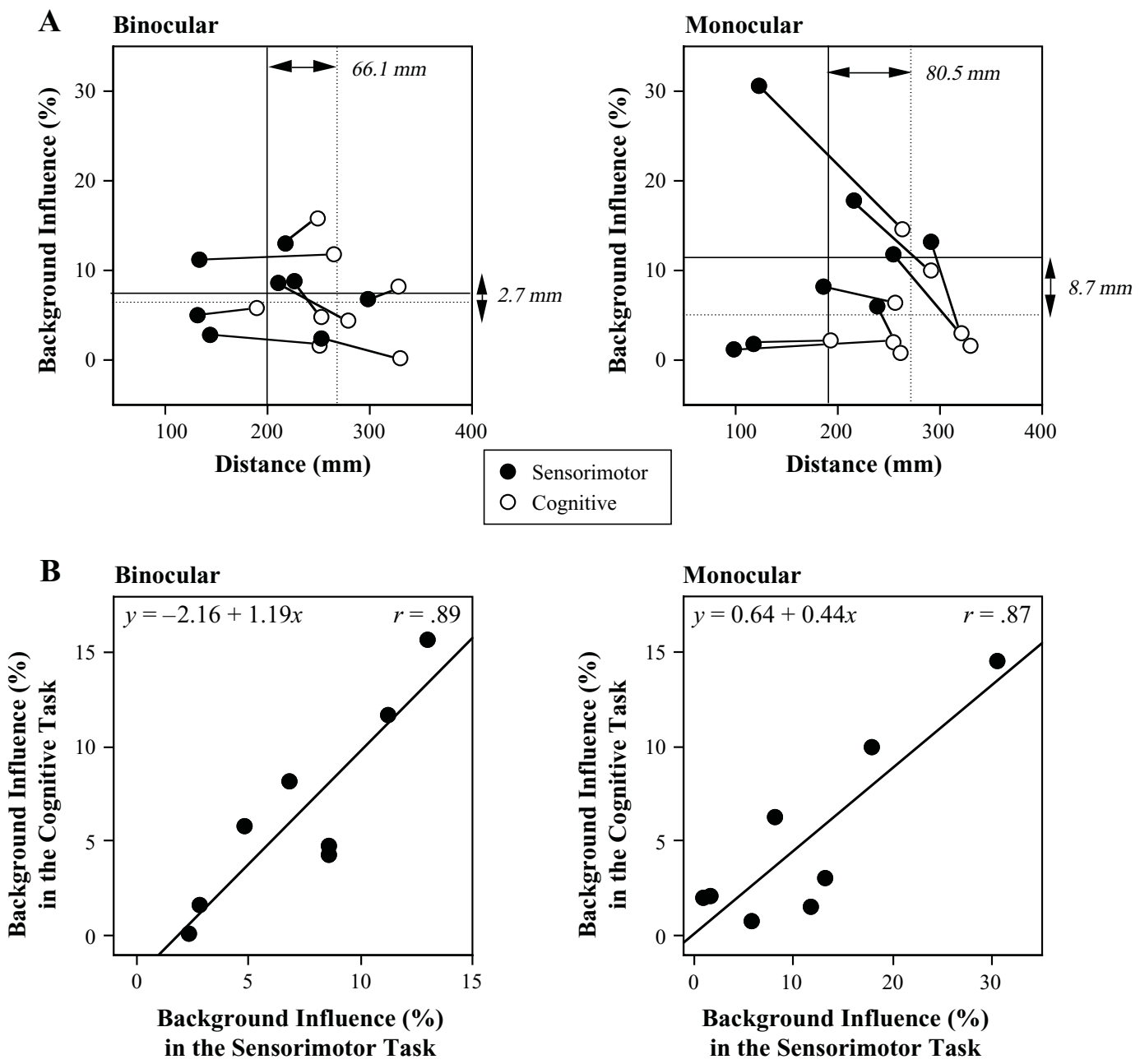

Figure 5. (A) Individual percentages of background influence in the cognitive and sensorimotor tasks for the binocular and monocular vision conditions. Individual results in the cognitive and sensorimotor tasks have been connected with solid lines. The horizontal solid line indicates the mean background influence in the sensorimotor task, whereas the horizontal dashed line indicates the mean background influence in the cognitive task. The vertical solid line indicates the mean pointing distance, whereas the vertical dashed line indicates the critical limit of what is reachable in the presence of a textured background. (B) Correlation between background influence in the sensorimotor task and that in the cognitive task. Linear regression equations and $r$ values are indicated within the graph. 
approximately on the same horizontal axis (Figure 5A). Data consistency was more obvious, however, in the binocular condition (the difference of the effect was only $2.7 \mathrm{~mm}$ for a gap of $66.1 \mathrm{~mm}$ between the pointing distance and the critical limit of what is reachable) than in the monocular condition (the difference of the effect was $8.7 \mathrm{~mm}$ for a gap of $80.5 \mathrm{~mm}$ between the pointing distance and the critical limit of what is reachable). The fact that structuring the workspace had a similar effect in the cognitive and sensorimotor tasks was confirmed by calculating the coefficient of regression $r$ measuring the degree of linearity between the percentage of background influence in the cognitive and sensorimotor tasks (Figure 5B). The $r$ coefficients were .89 and .87 in the binocular and the monocular vision conditions, respectively, and were statistically significant $[t(6)=4.78$ and $t(6)=4.32$, respectively; both $p$ s $<.01]$. However, the two $r$ coefficients were not statistically different (following $z$-score transform; $z=0.14, p>.05$ ). Furthermore, the slope (1.19) in the binocular condition was close to 1 and the intercept $(-2.16)$ was close to 0 , which was expected assuming that context information in a natural viewing condition influenced identically the cognitive and the sensorimotor distance codings of visual target.

\section{DISCUSSION}

The goal of the present study was to quantify the influence of context information on a spatial task entailing both cognitive and sensorimotor processing of the egocentric distance of a visual target. Our rationale was that, considering that context information (in the form of a textured background) influences cognitive and sensorimotor target coding similarly, the gain in distance performance of presenting the target together with the context should be of the same magnitude but opposite in direction. Previous studies have indeed demonstrated that visual objects are generally perceived as closer in sparse visual environments than in structured environments (Coello et al., 2000; Foley, 1991). Furthermore, it is acknowledged that the mere addition of a texture in the workspace is enough to improve distance coding (Coello, 2002; Magne $\&$ Coello, 2002). Thus, in a test of the sensorimotor system, movement amplitude was expected to increase in the presence of a textured background, whereas, in a test of the cognitive system, the distance corresponding to the critical limit below which visual targets are perceived as reachable was expected to decrease.

Data in the present study showed that in the sensorimotor task movement amplitude undershot the target by $46 \mathrm{~mm}$ in the absence of the textured background and increased by $17 \mathrm{~mm}$ when the textured background was available. By contrast, in the cognitive task the critical limit of what is reachable in darkness was situated $14 \mathrm{~mm}$ beyond the critical boundary specified by arm length, but it was $15 \mathrm{~mm}$ nearer in the presence of the textured background. The effects of structuring the workspace in the cognitive and sensorimotor tasks were thus of the same magnitude but opposite in direction, in agreement with our prediction. The assumption that context information affected cognitive and sensorimotor processing in similar ways was further supported by the highly significant linear relationship that we found when plotting the individual percentages of context influence in the cognitive task against those obtained in the sensorimotor task. In the most ecological situation - that is, the binocular vision condition - the coefficient of correlation measuring the degree of linearity of the relationship between the cognitive and sensorimotor percentages of context influence was high $(r=.89)$. This indicates that in the sensorimotor task the influence of the textured background was highly predictable from that observed in the cognitive task, and vice versa. Accordingly, it seems reasonable to assume that the influence of context information had a common origin in both tasks, and that this involves all of the participants.

The critical limit of what is reachable corresponded to about the actual limit of the reachability space (i.e., the maximum arm extension) in the presence of the textured background, whereas it was significantly farther in darkness. This outcome replicates previous findings and suggests that the critical limit of what is reachable is mainly a function of the capabilities of the observer's body (Bootsma et al., 1992; Carello et al., 1989; Rochat \& Wraga, 1997). In these studies, the intention to reach a target with the index finger depended on the distance of the target relative to the length of the arm. However, a consistent overestimation of reaching capabilities of about $10 \%$ was also reported. Such overestimation was thought to originate from people's everyday experience of reaching, which naturally requires multiple skeletal degrees of freedom, whereas experimental participants are generally tested in restricted postural situations that prevent natural body movement (Rochat \& Wraga, 1997). Indeed, when the limit of the reaching space was evaluated with no postural constraint (i.e., using the torso and the arm instead of merely the arm), the overestimation diminished (Carello et al., 1989). The fact that the overestimation was also smaller in the present study when the workspace was structured with the textured background suggests that perceptual factors, and not only postural ones, may interfere with the evaluation of the limit of what is reachable. Our data further suggest that impoverishing the visual scene produced a constriction of the visual space, with the consequence that visual targets were perceived as closer than they actually were (see also Bingham \& Pagano, 1998). Accordingly, the subjective limit of what is reachable exceeded the actual body capabilities with a bias of the same magnitude as that of the perceptual limit, but in the opposite direction. It is worth noting that in some of the previous studies that reported an overestimation of what is reachable, spatial performance in sparsely structured visual environments was analyzed. This is the case, for instance, in Carello et al.'s (1989) experiment, in which participants were asked to evaluate the reachability of visual targets presented on an uninformative black back- 
ground. Thus, the fact that the observed overestimation was slightly reduced but did not vanish when judgments were made in the absence of postural constraints suggests that it may originate from confounded factors.

The sensorimotor performance in the present experiment was also consistent with previous findings. It is indeed taken for granted that a visual target is generally undershot when presented in sparse visual conditions and without vision of the limb during action (Coello et al., 2000; Foley, 1991; Prablanc, Echallier, Jeannerod, \& Komilis, 1979). The perceptual nature of undershooting is supported by the tendency of the variations of peak velocity and movement time to mimic the variations of movement extent, and other studies have demonstrated that this does not involve calibration processes (Magne \& Coello, 2002). Because the proportion of time taken by the acceleration phase did not vary according to whether the textured background was available or not, the benefit gained from structuring the workspace can be better accounted for by improvement in distance perception than by optimized visuomotor transformations (Flanders, Helms Tillery, \& Soechting, 1992). Our data are thus in agreement with the assumption that presenting a visual target in an otherwise dark environment brings about a constriction of the whole visual space that induces misperception of target location, resulting in movement undershooting.

The fact that the effect of context was in the same direction in the monocular and binocular viewing conditions indicates that, in the presence of a structured environment, the visual system makes use of information available at the retinal level for distance coding. The lack of significant retinal information thus represents an unpropitious perceptual situation for accurate processing of distance, since it produces underestimation (Foley, 1991; Magne \& Coello, 2002). The tendency in darkness for spatial performance to be worse in the monocular than in the binocular vision condition (in terms of underestimation and variability) suggests, nonetheless, that binocular nonretinal sources of information (e.g., vergence) can contribute to distance coding, notably in impoverished visual conditions (see also Bingham \& Pagano, 1998; Coello et al., 2000). However, the similar improvement in the presence of the textured background in the monocular and binocular viewing conditions establishes retinal signals as a prevailing source of information for distance coding in a structured environment.

Current dominant theory concerning the organization of the visual system suggests that different forms of spatial coding (i.e., cognitive and sensorimotor) occur according to whether the physical characteristics of visual stimuli are to be reported or used to guide the motor act (Bridgeman, 1991; Milner \& Goodale, 1995; Rossetti, 1998). According to this perspective, the visual system for guidance of action uses a sensorimotor coding that is tied to an egocentric frame of reference, whereas for overt description a quite different, context-based (allocentric) form of spatial coding would be employed. With the latter, the location of a visual stimulus is thought to be de- termined from an interaction between the target and the surrounding environmental cues principally because the latter are supposed to be stable (Bridgeman, 2000; Milner \& Goodale, 1995; Paillard, 1987; Rossetti, 1998). From a physiological standpoint, the distinct influence of context information was supposed to be mediated through two main neural tracts conveying the visual information that exits the striate cortex (Goodale \& Milner, 1992; Milner $\&$ Goodale, 1995). The dorsal stream, projecting to regions in the posterior parietal lobe, is assumed to serve as support for immediate guided action, whereas the ventral stream, reaching areas within the inferior temporal cortex, is thought to allow object identification. In agreement with previous statements, only the ventral stream is supposed to be influenced by context information because it deals with relative positions. In conflict with this perspective, data in the present study argue against a radical separation between cognitive and sensorimotor visual functions on the basis of the principle that only the cognitive system is context sensitive. The provision of the textured background in the workspace resulted in a decrease of the subjective reaching area of about $15 \mathrm{~mm}$, whereas the amplitude of the pointing movement increased by about the same amount. As we have already mentioned, this reverse effect can be accounted for by the simple fact that visual space shrank in the absence of meaningful environmental cues. However, this perceptual effect should occur at a stage of visual processing that is common to both the cognitive and the sensorimotor visual systems.

Accordingly, the analogy between the cognitive and sensorimotor uses of visual information and the existence of independent cortical pathways within the visual brain requires some reevaluation. First, the congruent pattern of results in the cognitive and sensorimotor tasks could be attributed to the fact that the two visual systems conveying spatial information for different purposes are both influenced by context information. However, for all the participants the strict symmetrical influence of the textured background in the two tasks did not argue in favor of a different influence of context information within two independent visual streams. One could wonder, moreover, what would be the benefit for the visual system of having two independent pathways dealing with identical spatial processing. This also rules out the possibility that context information influenced motor planning but not control process, allowing on-line minimization of the spatial error of the movement (the planning-control model; Glover, 2004; Glover \& Dixon, 2002). An alternative would be to consider that the processing of context information remains the prerogative of the ventral stream, which can influence the sensorimotor system in some circumstances. Interpreting the data accordingly causes one to regard distance and direction parameters as specified through independent visual streams. As a consequence, distance coding would be affected by context information simply because this spatial dimension would be preferentially computed within the ventral stream. However, the previous observation with visual illusions - that is, that context 
effect can interfere to the same extent with both cognitive and sensorimotor directional coding when a delay of a few seconds is introduced before response - did not support this interpretation (see, e.g., Bridgeman's 1991 demonstration with the induced Roelofs effect). Furthermore, the influence of context in the sensorimotor task with delayed responses was thought to account mainly for the temporal constraints associated with the transfer and maintenance of information within the ventral and dorsal streams (Rossetti, 1998; Rossetti \& Pisella, 2002).

Thus, the existence of early cross-talk between the ventral and dorsal parts of the visual system as a mechanism for integration of context information represents the best alternative for accounting for the present data. Since it is acknowledged from brain lesion studies that the cognitive and sensorimotor tasks involve the ventral and the dorsal pathways of the visual system, respectively (Milner \& Goodale, 1995), the observation that visual context can influence cognitive and sensorimotor distance coding similarly suggests that the two visual pathways interact at some stage of visual processing, at least within an undamaged brain (Coello et al., 2003; Humphreys, Riddoch, Forti, \& Ackroyd, 2004; Rossetti \& Pisella, 2002). It was hypothesized that such an interaction could occur within the inferior parietal lobule (IPL; Binkofski et al., 1998), which may constitute the locus of a third visual system (Boussaoud, Ungerleider, \& Desimone, 1990; Glover, 2004; Rizzolatti \& Matelli, 2003) involved in both visuomotor behavior and visual-dependent cognitive tasks. This area received projection from V1 as well as from the temporal lobe (Boussaoud et al., 1990), which provides information about the structure of the environment (e.g., depth cues; Carey, Dijkerman, \& Milner, 1998) as well as about semantic context (e.g., tool use; Glover, 2004). It also receives projection from other areas, such as the superior parietal lobe and the frontal and somatosensory cortices. The implication of this parietal subsystem in action planning has been repeatedly suggested (Deiber, Ibañez, Sadato, \& Hallett, 1996; Glover, 2004; Grafton, Arbib, Fadiga, \& Rizzolatti, 1996; Mattingley, Husain, Rorden, Kennard, \& Driver, 1998; Rushworth, Krams, \& Passingham, 2001). Similarly, increased activity was found in IPL when a more cognitive task, such as internal action simulation (Decety et al., 1994), was performed, which might be involved in judging what is reachable and what is not (Fischer, 2000). Thus, viewing the visual system as a more interactive network enabling communication between the different visual processes is perhaps more appropriate than the perception-action dichotomy (Binsted \& Elliott, 1999; Goodman \& Anderson, 1989; Humphreys et al., 2004). It is worth mentioning, however, that in the present study the cognitive task showed no sign of binocular-monocular effects, but the sensorimotor task did. Furthermore, the slope of the regressions relating the percentage of background influence in the cognitive task to that in the sensorimotor task is substantially less than one with monocular view- ing. One interpretation of this could be that there was a distinctive effect of textured background in pointing and spatial judgment under monocular viewing, ${ }^{1}$ which suggests that monocular vision is not equivalent to binocular vision (Bingham \& Pagano, 1998). However, spatial performance when a textured background was provided was similar in the cognitive and sensorimotor tasks in both the monocular and binocular viewing conditions. Furthermore, the coefficient of correlation between the background influence in the cognitive and sensorimotor tasks was high in the monocular condition (.87) and not statistically different from that in the binocular condition (.89). Thus, perception and action may share similar visual resources, obviously within a common visual stream in the presence of a structured environment. However, in the absence of relevant visual input (i.e., under spatial uncertainty), distance evaluation for sensorimotor purposes might rely more heavily on extraretinal signals, which are known to be inaccurate in darkness (Magne \& Coello, 2002; Tresilian et al., 1999), whereas cognitive judgment might favor pictorial depth cues (e.g., relative size or illumination), for which the ventral stream is supposed to play an important role (Dijkerman, Milner, \& Carey, 1996; Mon-Williams, Tresilian, McIntosh, \& Milner, 2001).

In conclusion, the present findings argue against a radical separation between perception and action on the basis of a differential influence of visual context. Whether performance is context dependent or not seems to be predictable not only from the characteristics of the output (Milner \& Goodale, 1995), but also from the (relative vs. absolute) nature of spatial processing, as is suggested in the present study and in line with previous statements (Coello et al., 2003; Vishton, Rea, Cutting, \& Nuñez, 1999).

\section{REFERENCES}

Allport, D. A. (1989). Visual attention. In M. I. Posner (Ed.), The foundations of cognitive science (pp. 658-664). Cambridge, MA: MIT Press.

BInGHaM, G. P. (1993). Perceiving the size of trees: Form as information about scale. Journal of Experimental Psychology: Human Perception \& Performance, 19, 1139-1161.

Bingham, G. P., \& Pagano, C. C. (1998). The necessity of a perception/ action approach to definite distance perception: Monocular distance perception to guide reaching. Journal of Experimental Psychology: Human Perception \& Performance, 24, 145-168.

Binkofski, F., Dohle, C., Posse, S., Stephan, K. M., Hefter, H. Seitz, R. J., \& Freund, H. J. (1998). Human anterior intraparietal area subserves prehension: A combined lesion and functional MRI activation study. Neurology, 50, 1253-1259.

Binsted, G., \& ElliotT, D. (1999). Ocular perturbations and retinal/ extraretinal information: The coordination of saccadic and manual movements. Experimental Brain Research, 127, 193-206.

Bootsma, R. J., BaKker, F. C., VAn Snippenberg, F. J., \& TDlohreg, C. W. (1992). The effects of anxiety on perceiving the reachability of passing objects. Ecological Psychology, 4, 1-16.

Boussaoud, D., Ungerleider, L. G., \& Desimone, R. (1990). Pathways for motion analysis: Cortical connections of the medial superior temporal and fundus of the superior temporal visual areas in the macaque. Journal of Comparative Neurology, 296, 462-495.

BRIDGEMAN, B. (1991). Separate visual representations for perception 
and for visually guided behavior. In S. R. Ellis, M. K. Kaiser, \& A. C. Grunwald (Eds.), Pictorial communication in virtual and real environments (pp. 316-327). London: Taylor \& Francis.

BRIDGEMAN, B. (2000). Interactions between vision for perception and vision for behavior. In Y. Rossetti \& A. Revonsuo (Eds.), Beyond dissociation: Interaction between dissociated implicit and explicit processing (pp. 17-40). Amsterdam: Benjamins.

Bridgeman, B., Peery, S., \& Anand, S. (1997). Interaction of cognitive and sensorimotor maps of visual space. Perception \& Psychophysics, 59, 456-469.

Carello, C., Grosofsky, A., Reichel, F. D., Solomon, H. Y., \& TurVEY, M. T. (1989). Visually perceiving what is reachable. Ecological Psychology, 1, 27-54.

Carey, D. P., Dijkerman, H. C., \& Milner, A. D. (1998). Perception and action in depth. Consciousness \& Cognition, 7, 438-453.

Coello, Y. (2002). Role of environmental cues in position coding. In J. A. Da Silva, E. H. Matsushima, \& N. P. Ribeiro-Filho (Eds.), Fechner Day 2002 (pp. 252-258). Rio de Janeiro: Legis Summa.

Coello, Y., \& Grealy, M. A. (1997). Effect of size and frame of visual field on the accuracy of an aiming movement. Perception, 26, 287-300.

Coello, Y., Magne, P., \& Plenacoste, P. (2000). The contribution of retinal signal to the specification of target distance in a visuo-manual task. Current Psychology Letters, 3, 75-89.

Coello, Y., Richaud, S., Magne, P., \& Rossetti, Y. (2003). Vision for spatial perception and vision for action: A dissociation between the left-right and near-far dimensions. Neuropsychologia, 41, 622633.

Coello, Y., \& Rossetti, Y. (2004). Planning and controlling action in a structured environment: Visual illusion without dorsal stream. Behavioral \& Brain Sciences, 27, 29-31.

Conti, P., \& Beaubaton, D. (1980). Role of structured visual field and visual reafference in accuracy of pointing movements. Perceptual \& Motor Skills, 50, 239-244.

Decety, J., Perani, D., Jeannerod, M., Bettinardi, V., Tadary, B., Woods, R., ET AL. (1994). Mapping motor representations with positron emission tomography. Nature, 371, 600-602.

Deiber, M.-P., Ibañez, V., Sadato, N., \& Hallett, M. (1996). Cerebral structures participating in motor preparation in humans: A positron emission tomography study. Journal of Neurophysiology, 75, 233-247.

Desimone, R., \& Duncan, J. (1995). Neural mechanisms of selective visual attention. Annual Review of Neuroscience, 18, 193-222.

Dijkerman, H. C., Milner, A. D., \& Carey, D. P. (1996). The perception and prehension of objects oriented in the depth plane: I. Effects of visual form agnosia. Experimental Brain Research, 112, 442-451.

Fischer, M. H. (2000). Estimating reachability: Whole body engagement or postural stability? Human Movement Science, 19, 297-318.

Flanders, M., Helms Tillery, S. I., \& Soechting, J. F. (1992). Early stages in sensorimotor transformation. Behavioral \& Brain Sciences, 15, 309-362.

Foley, J. M. (1991). Stereoscopic distance perception. In S. R. Ellis, M. K. Kaiser, \& A. C. Grunwald (Eds.), Pictorial communication in virtual and real environments (pp. 558-566). New York: Taylor \& Francis.

Foley, J. M., \& HeLD, R. (1972). Visually directed pointing as a function of target distance, direction, and available cues. Perception \& Psychophysics, 12, 263-268.

Franz, V. H., Gegenfurtner, K. R., Bülthoff, H. H., \& Fahle, M. (2000). Grasping visual illusions: No evidence for a dissociation between perception and action. Psychological Science, 11, 20-25.

GLOver, S. (2004). Separate visual representations in the planning and control of action. Behavioral \& Brain Sciences, 27, 3-78.

Glover, S., \& Dixon, P. (2002). Dynamic effects of the Ebbinghaus illusion in grasping: Support for a planning/control model of action. Perception \& Psychophysics, 64, 266-278.

Goodale, M. A., \& Milner, A. D. (1992). Separate visual pathways for perception and action. Trends in Neurosciences, 15, 20-35.

Goodman, S. J., \& Anderson, R. A. (1989). Microstimulation of a neural network model for visually guided saccades. Journal of Cognitive Neuroscience, 1, 317-327.
Grafton, S. T., Arbib, M., Fadiga, L., \& Rizzolatti, G. (1996). Localization of grasp representations in humans by positron emission tomography: II. Observation compared with imagination. Experimental Brain Research, 112, 103-111.

Grealy, M. A., Coello, Y., \& Heffernan, D. (2003). Apparent motion cues distort object localisation in egocentric space. Experimental Brain Research, 150, 356-362.

Humphreys, G. W., Riddoch, M. J., ForTI, S., \& AcKroyd, K. (2004). Action influences spatial perception: Neuropsychological evidence. Visual Cognition, 11, 401-427.

Jeannerod, M., \& Rossetti, Y. (1993). Visuomotor coordination as a dissociable function: Experimental and clinical evidence. Baillière's Clinical Neurology, 2, 439-460.

KossLYN, S. M. (1994). Image and brain: The resolution of the imagery debate. Cambridge, MA: MIT Press.

Magne, P., \& CoELlo, Y. (2002). Retinal and extra-retinal contribution to position coding. Behavioural Brain Research, 136, 277-287.

Mattingley, J. B., \& Driver, J. (1997). Distinguishing sensory and motor deficits after parietal damage: An evaluation of response selection biases in unilateral neglect. In P. Thier \& H. O. Karnath (Eds.), Parietal lobe contributions to orientation in $3 D$ space (pp. 309-337). Berlin: Springer.

Mattingley, J. B., Husain, M., Rorden, C., Kennard, C., \& DrIVER, J. (1998). Motor role of human inferior parietal lobe revealed in unilateral neglect patients. Nature, 392, 179-182.

Milner, A. D., \& Goodale, M. A. (1995). The visual brain in action. Oxford: Oxford University Press.

Mon-Williams, M., Tresilian, J. R., Mcintosh, R. D., \& Milner, A. D. (2001). Monocular and binocular distance cues: Insights from visual form agnosia I (of III). Experimental Brain Research, 139, 127-136.

Pagano, C. C., \& Bingham, G. P. (1998). Comparing measures of monocular distance perception: Verbal and reaching errors are not correlated. Journal of Experimental Psychology: Human Perception \& Performance, 24, 1037-1051.

PaILlaRd, J. (1987). Cognitive versus sensorimotor encoding of spatial information. In P. Ellen \& C. Thinus-Blanc (Eds.), Cognitive processes and spatial orientation in animal and man (pp. 43-77). Dordrecht: Nijhoff.

Prablanc, C., Echallier, J. E., Jeannerod, M., \& Komilis, E. (1979). Optimal response of eye and hand motor systems in pointing at a visual target: II. Static and dynamic visual cues in the control of hand movement. Biological Cybernetics, 35, 183-187.

Rizzolatti, G., \& Matelli, M. (2003). Two different streams form the dorsal visual system: Anatomy and functions. Experimental Brain Research, 153, 146-157.

Rochat, P., \& Wraga, M. (1997). An account of the systematic error in judging what is reachable. Journal of Experimental Psychology: Human Perception \& Performance, 23, 199-212.

RossetTI, Y. (1998). Implicit short-lived motor representation of space in brain-damaged and healthy subjects. Consciousness \& Cognition, 7, 520-558

Rossetti, Y., \& Pisella, L. (2002). Several "vision for action" systems: A guide to dissociating and integrating dorsal and ventral functions. In W. Prinz \& B. Hommel (Eds.), Common mechanisms in perception and action: Attention and performance XIX (pp. 62-119). Oxford: Oxford University Press.

Rushworth, M. F., Krams, M., \& Passingham, R. E. (2001). The attentional role of the left parietal cortex: The distinct lateralization and localization of motor attention in the human brain. Journal of Cognitive Neuroscience, 13, 698-710.

SchneIDER, G. E. (1969). Two visual systems. Science, 163, 895-902.

TipPer, S. P., LorTiE, C., \& BaYlis, G. C. (1992). Selective reaching: Evidence for action-centered attention. Journal of Experimental Psychology: Human Perception \& Performance, 18, 891-905.

Tresilian, J. R., Mon-Williams, M., \& Kelly, B. M. (1999). Increasing confidence in vergence as a cue to distance. Proceedings of the Royal Society of London: Series B, 266, 39-44.

Trevarthen, C. B. (1968). Two mechanisms of vision in primates. Psychologische Forschung, 31, 299-337. 
Ungerleider, L., \& MishKin, M. (1982). Two cortical visual systems. In D. J. Ingle, M. A. Goodale, \& R. J. W. Mansfield (Eds.), Analysis of visual behavior (pp. 549-586). Cambridge, MA: MIT Press.

Vishton, P. M., ReA, J. G., Cutting, J. E., \& NuñEz, L. N. (1999). Comparing effects of the horizontal-vertical illusion on grip scaling and judgment: Relative versus absolute, not perception versus action. Journal of Experimental Psychology: Human Perception \& Performance, 25, 1659-1672.

WatT, S. J., Bradshaw, M. F., \& Rushton, S. K. (2000). Field of view affects reaching, not grasping. Experimental Brain Research, 135, $411-416$

\section{NOTE}

1. We are grateful to one of the reviewers, Peter Dixon, for his constructive and thoughtful comments and for having suggested this possible interpretation of our data.

(Manuscript received October 14, 2003 ; revision accepted for publication March 21, 2005.) 